\title{
DIAMETERS OF HOMOGENEOUS SPACES
}

\author{
Michael H. Freedman, Alexei Kitaev, and Jacob Lurie
}

\begin{abstract}
Let $G$ be a compact connected Lie group with trivial center. Using the action of $G$ on its Lie algebra, we define an operator norm ||$_{G}$ which induces a bi-invariant metric $d_{G}(x, y)=\left|\operatorname{Ad}\left(y x^{-1}\right)\right|_{G}$ on $G$. We prove the existence of a constant $\beta \approx .12$ (independent of $G$ ) such that for any closed subgroup $H \subsetneq G$, the diameter of the quotient $G / H$ (in the induced metric) is $\geq \beta$.
\end{abstract}

\section{Introduction}

Finding a lower bound to the (operator norm) diameter of homogeneous spaces $G / H, G$ compact is a natural geometric problem. It can also be motivated by considering quantum computation. In standard models $[\mathrm{NC}]$ the state space of a (theoretical) quantum computer is a Hilbert space with a tensor decomposition, $\left(\mathbb{C}^{2}\right)^{\otimes n}$. A "gate" is a local unitary operation acting on a small number, perhaps two, tensor factors (and as the identity on the remaining factors). One often wonders if a certain set of local gates is "universal" meaning that the closed subgroup $H$ they generate satisfies $U(1) H=U\left(2^{n}\right)$. We produce a constant $\beta \approx .12$ so that diam $U\left(2^{n}\right) / U(1) H<\beta$ implies universality, where diameter is to be computed in the operator norm. This norm is well-suited here because it is stable under $\otimes_{\mathrm{id}}$.

Because the operator norm is bi-invariant it suffices to check that every element $b$ in the ball of radius $2 \beta$ about the identity of $S U\left(2^{n}\right)$ has $\operatorname{Ball}_{\beta}(b) \cap H \neq \emptyset$. In principle this leads to an algorithm to test if a gate set is universal. Such an algorithm will be exponentially slow in $\mathrm{n}$. But often it is assumed that identical gates can be applied on any pair of $\mathbb{C}^{2}$ factors; in this case universality for $n=2$ is sufficient to imply universality for all $n$.

Let $G$ be a compact Lie group with trivial center. The semisimplicity of $G$ implies that the (negative of the) Killing form is a natural positive-definite, bi-invariant inner product on the Lie algebra $\mathfrak{g}$ of $G$. We let $\|x\|_{\mathfrak{g}}$ denote the induced (Euclidean) norm on $\mathfrak{g}$. We use this to define the operator norm on $G$ as follows:

$$
|g|_{G}=\sup _{\|y\|_{\mathfrak{g}}=1}\left|\angle\left(y, \operatorname{Ad}_{g} y\right)\right|
$$

where $\angle\left(y, \operatorname{Ad}_{g} y\right)$ denotes the usual Euclidean angle between the vectors $y$ and $\operatorname{Ad}_{g} y$, normalized so that it lies in the interval $[-\pi, \pi]$. Since angles between

Received June 21, 2002.

Revised version received November 4, 2002. 
vectors in a Euclidean space obey a triangle inequality, we deduce the inequality $|g h|_{G} \leq|g|_{G}+|h|_{G}$. It is also clear that $|g|_{G}=0$ if and only if $\operatorname{Ad}_{g}$ is the identity, which implies that $g$ is the identity since the adjoint action of $G$ is faithful up to the center of $G$, and we have assumed that the center of $G$ is trivial.

We define a distance on $G$ by the formula $d_{G}\left(g, g^{\prime}\right)=\left|g^{-1} g^{\prime}\right|_{G}$. It is easy to check that this defines a bi-invariant metric on $G$, where all distances are bounded above by $\pi$. Note that $d_{G}$ is continuous on $G$, hence there is a continuous bijection from $G$ with its usual topology to $G$ with the topology induced by $d_{G}$. Since the source is compact and the target Hausdorff (this fails if $G$ has nontrivial center, since the operator norm of a central element is equal to zero), we deduce that the metric $d_{G}$ determines the usual topology on $G$. For any closed subgroup $H$ of $G$, the homogeneous space $G / H$ inherits a quotient metric given by the formula

$$
d_{G / H}(p, q)=\inf d_{G}(\widetilde{p}, \widetilde{q})=\inf _{g p=q}|g|_{G}
$$

where the first infimum is taken over all pairs $\widetilde{p}, \widetilde{q} \in G$ lifting the pair $p, q \in G / H$. Note that if $H$ is contained in $H^{\prime}$, then the diameter of $G / H$ is at least as large as that of $G / H^{\prime}$. We are now in a position to state the main result:

Theorem 1. Let $G$ be a compact connected Lie group with trivial center and $H \varsubsetneqq G$ a proper compact subgroup of $G$. Then the diameter of $G / H$ with respect to the metric $d_{G / H}$ is no smaller than $\beta$, where $\beta$ is the smallest real solution to the transcendental equation $\cos ^{2}(\alpha-\beta)+\sin ^{2}(\alpha-\beta) \sin (\beta)=\cos (4 \beta)$ and $\cos (\alpha)=\frac{7}{8}$.

One can estimate that the constant $\beta$ is approximately .124332.

Example 2. Consider the case where $G=H \times H$ is a product, and $H$ is embedded diagonally. Choose an element $h \in H$ with $|h|_{H}=\pi$ (such an element exists in any nontrivial one parameter subgroup). Then in $H \times H$, the distance $d_{H \times H}\left(h \times 0, h^{\prime} \times h^{\prime}\right)$ is equal to the larger of $d_{H}\left(h, h^{\prime}\right)$ and $d_{H}\left(h^{\prime}, e\right)$. By the triangle inequality, this distance is at least $\frac{\pi}{2}$. It follows that the diameter of $G / H$ is at least $\frac{\pi}{2}$.

\section{Remarks.}

(1) For any orthogonal representation $\tau: G \rightarrow O(V)$ of a group $G$, we can define an operator norm on $G$ with respect to $V$ :

$$
|g|_{G, \tau}=\sup _{\|v\|=1}|\angle(v, g v)|
$$

This construction has the following properties:

- If $V$ is the complex plane $\mathbb{C}$, and $g \in G$ acts by multiplication by $e^{i \alpha}$ where $-\pi \leq \alpha \leq \pi$, then $|g|_{G, \tau}=|\alpha|$.

- Given any subgroup $H \subseteq G$, the restriction of $\|_{G, \tau}$ to $H$ is equal to ${ }_{|H, \tau| H}$. 
- The operator norm associated to a direct sum of representations $\tau_{i}$ of $G$ is the supremum of the operator norms associated to the representations $\tau_{i}$.

- In particular, the operator norm on $G$ associated to a representation $V$ is identical with the operator norm on $G$ associated to the complexification $V \otimes_{\mathbb{R}} \mathbb{C}$ (with its induced Hermitian structure).

- To evaluate $|g|_{G, \tau}$, we can replace $G$ by the subgroup generated by $g$ and $V$ by its complexification, which decomposes into one-dimensional complex eigenspaces under the action of $g$. We deduce that $|g|_{G, \tau}$ is the supremum of $\left|\log \lambda_{j}\right|$, where $\left\{\lambda_{j}\right\}$ is the set of eigenvalues for the action of $g$ on $V$ (and the logarithms are chosen to be of absolute value $\leq \pi)$.

(2) The reader may be curious about the diameter of $G / H$ relative to the Riemannian quotient of the Killing metric $d_{K}$. If we let $N$ denote the dimension of $\mathfrak{g}$, then we have

$$
d \leq d_{K} \leq \frac{3 N^{\frac{1}{2}} d}{2}
$$

(3) We ask if the quotient $S O(3) / I$ is the homogenous space of smallest diameter, where $I \simeq A_{5}$ denotes the symmetry group of the icosahedron.

(4) We wonder if there is a similar universal lower bound to the diameter of double coset spaces $K \backslash G / H, G$ as above, $K, H \subset G$ closed subgroups. Our method does not apply directly.

(5) Although suggested by a modern subject the theorem could easily have been proved a hundred years ago and in fact may have been (or may be) known.

\section{Small Subgroups}

Throughout this section, $G$ shall denote a compact, connected Lie group with trivial center. We give a quantitative version of the principle that discrete subgroups of $G$ generated by "sufficiently small" elements are automatically abelian. We will use this in the proof of Theorem 1 in the case where $H$ is discrete. We will need to understand the operator norm on $G$ a bit better. To this end, we introduce the operator norm

$$
|x|_{\mathfrak{g}}=\sup _{\|y\|_{\mathfrak{g}}=1}\|[x, y]\|_{\mathfrak{g}}
$$

on the Lie algebra $\mathfrak{g}$ of $G$. This is a $G$-invariant function on $\mathfrak{g}$, so we can unambiguously define the operator norm of any tangent vector to the manifold $G$ by transporting that tangent vector to the origin (via left or right translation) and then applying $x \mapsto|x|_{\mathfrak{g}}$. The operator norm on $\mathfrak{g}$ is related to the operator norm on $G$ by the following: 
Lemma 3. The exponential map $x \mapsto \exp (x)$ induces a bijection between $\mathfrak{g}_{0}=$ $\left\{x \in \mathfrak{g}:|x|_{\mathfrak{g}}<\frac{2 \pi}{3}\right\}$ and $G_{0}=\left\{g \in G:|g|_{G}<\frac{2 \pi}{3}\right\}$. This bijection preserves the operator norms.

Proof. First, we claim that the map $x \mapsto \exp (x)$ does not increase the operator norm. This follows from the fact that the eigenvalues of $\exp (x)$ have the form $\exp (\kappa)$, where $\kappa$ is an eigenvalue of $x$. It follows that the exponential map sends $\mathfrak{g}_{0}$ into $G_{0}$. Choose $g \in G_{0}$, and fix a maximal torus $T$ containing $g$. Let $\mathfrak{t}$ be the Lie algebra of $T$. Decompose $\mathfrak{g} \otimes_{\mathbb{R}} \mathbb{C}$ into eigenspaces for the action of $T$ : $\mathfrak{g} \otimes_{\mathbb{R}} \mathbb{C}=\mathfrak{t} \otimes_{\mathbb{R}} \mathbb{C} \oplus \bigoplus_{\alpha} \mathfrak{g}_{\alpha}$. The element $g$ acts by an eigenvalue $\Lambda(\alpha)$ on each nonzero eigenspace $\mathfrak{g}_{\alpha}$. Since $g$ is an orthogonal transformation, we may write $\Lambda(\alpha)=e^{i \lambda(\alpha)}$. Since $g \in G_{0}$, it is possible to choose the function $\lambda$ so that $-\frac{2 \pi}{3}<\lambda(\alpha)<\frac{2 \pi}{3}$ for each root $\alpha$. This determines the function $\lambda$ uniquely. Choose a system $\Delta$ of simple roots, and let $x$ be the unique element of $\mathfrak{t}$ such that $\alpha(x)=\lambda(\alpha)$ for each $\alpha \in \Delta$. It follows immediately that $\exp (x)=g$ (since $G$ has trivial center). To show that $x \in \mathfrak{g}_{0}$, we need to show that $|\alpha(x)|<\frac{2 \pi}{3}$ for all roots $\alpha$. For this, it will suffice to prove that $\alpha(x)=\lambda(\alpha)$ for all roots $\alpha$. The uniqueness of $\lambda$ implies immediately that $\lambda(-\alpha)=-\lambda(\alpha)$. Thus, it will suffice to prove that the equation $\alpha(x)=\lambda(\alpha)$ holds when $\alpha$ is positive (with respect to the root basis $\Delta$ ). Since the equation is known to hold whenever $\alpha \in \Delta$, it will suffice to prove that $\alpha(x)=\lambda(\alpha), \beta(x)=\lambda(\beta)$ implies

$$
(\alpha+\beta)(x)=\lambda(\alpha+\beta) .
$$

In other words, we need to show that the quantity

$$
\epsilon=\lambda(\alpha+\beta)-\lambda(\alpha)-\lambda(\beta)
$$

is equal to zero. By construction, $|\epsilon|<2 \pi$. On the other hand, since $\Lambda(\alpha) \Lambda(\beta)=$ $\Lambda(\alpha+\beta)$, we deduce that $e^{i \epsilon}=1$, so that $\epsilon$ is an integral multiple of $2 \pi$. It follows that $\epsilon=0$, as desired. It is clear from the construction that $|x|_{\mathfrak{g}}=|g|_{G}$. To complete the proof, we need to show that $g$ has no other logarithms lying in $\mathfrak{g}_{0}$. This follows from the fact that any unitary transformation (in particular, the adjoint action of $g$ on $\mathfrak{g}$ ) which does not have -1 as an eigenvalue has a unique logarithm whose eigenvalues are of absolute value $<\pi$.

Lemma 4. Let $p:[0,1] \rightarrow G$ be a smooth function with $p(0)$ equal to the identity of $G$. Then $|p(1)|_{G} \leq \int_{0}^{1}\left|p^{\prime}(t)\right|_{\mathfrak{g}} d t$.

Proof. For $N$ sufficiently large, we can write $p\left(\frac{i+1}{N}\right)=p\left(\frac{i}{N}\right) \exp \left(\frac{x_{i}}{N}\right)$, where $x_{i}$ is approximately equal to the derivative of $p$ at $\frac{i}{N}$. Thus, as $N$ goes to $\infty$, the average $\frac{\left|x_{0}\right|_{\mathfrak{g}}+\ldots+\left|x_{N-1}\right|_{\mathfrak{g}}}{N}$ converges to the integral on the right hand side of the desired inequality. By the triangle inequality, it will suffice to prove that $\left|p\left(\frac{i}{N}\right)^{-1} p\left(\frac{i+1}{N}\right)\right|_{G} \leq\left|\frac{\left|x_{i}\right|_{\mathfrak{g}}}{N}\right|_{\mathfrak{g}}$. If $N$ is sufficiently large, then this follows immediately from Lemma 3 . 
Remark 5. The metric $d_{G}$ on $G$ is not necessarily a path metric: given $g, h \in G$, there does not necessarily exist a path in $G$ having length equal to $d_{G}(g, h)$. However, it follows from Lemma 3 that $d_{G}$ is a path metric locally on $G$. The length of a (smooth) path can be obtained by integrating the operator norm of the derivative of a path. Replacing $d_{G}$ by the associated path metric only increases distances, so that Theorem 1 remains valid for the path metric associated to $d_{G}$. This modified version of Theorem 1 makes sense (and remains true) for compact Lie groups $G$ with finite center.

We can now proceed to the main result of this section. Let $\alpha$ denote the smallest positive real number satisfying $\cos (\alpha)=\frac{7}{8}$.

Theorem 6. Let $H \subset G$ be a discrete subgroup. Let $h, k \in H$ and suppose $|h|_{G}<\frac{\pi}{2},|k|_{G}<\alpha$. Then $[h, k]=1$.

Proof. We define a sequence of elements of $G$ by recursion as follows: $h_{0}=h$, $h_{n+1}=\left[h_{n}, k\right]$. Let $C$ satisfy the equation $\frac{C^{2}}{4}=2-2 \cos |k|_{G}$. Then the assumption on $k$ ensures that $C<1$. Our first goal is to prove that the operator norm of the sequence $\left\{h_{n}\right\}$ obeys the estimate $\left|h_{n}\right|_{G}<C^{n} \frac{\pi}{2}$. For $n=0$, this is part of our hypothesis. Assuming that the estimate $\left|h_{n}\right|_{G}<C^{n} \frac{\pi}{2}$ is valid, we can use Lemma 3 to write $h_{n}=\exp (x),|x|_{\mathfrak{g}}<C^{n} \frac{\pi}{2}$. Now define $p(t)=$ $[\exp (t x), k]$, so that $p(0)=1$ and $p(t)=h_{n+1}$. Using Lemma 4 , we deduce that $\left|h_{n+1}\right|_{G} \leq \int_{0}^{1}\left|p^{\prime}(t)\right|_{\mathfrak{g}} \leq \sup _{t}\left|p^{\prime}(t)\right|_{\mathfrak{g}}$. On the other hand, the vector $p^{\prime}(t)$ can be written as a difference

$$
R_{p(t)} x-L_{\exp (t x) k \exp (-t x)} R_{k^{-1}} x
$$

where $R_{g}$ and $L_{g}$ denote left and right translation by $g$. We obtain

$$
\begin{aligned}
\left|p^{\prime}(t)\right|_{\mathfrak{g}} & =\left|x-\operatorname{Ad}_{\exp (t x) k \exp (-t x)} x\right|_{\mathfrak{g}} \\
& =\left|\operatorname{Ad}_{\exp (-t x)} x-\operatorname{Ad}_{k \exp (-t x)} x\right|_{\mathfrak{g}} \\
& =\left|x-\operatorname{Ad}_{k} x\right|_{\mathfrak{g}} \\
& =\sup _{\|y\|_{\mathfrak{g}}=1}\left\|\left[x-\operatorname{Ad}_{k} x, y\right]\right\|_{\mathfrak{g}} \\
& \leq \sup _{\|y\|_{\mathfrak{g}}=1}\left(\left\|[x, y]-\operatorname{Ad}_{k}[x, y]\right\|_{\mathfrak{g}}+\left\|\operatorname{Ad}_{k}[x, y]-\left[\operatorname{Ad}_{k} x, y\right]\right\|_{\mathfrak{g}}\right) \\
& \leq \sup _{\|y\|_{\mathfrak{g}}=1}\left\|[x, y]-\operatorname{Ad}_{k}[x, y]\right\|_{\mathfrak{g}}+\sup _{\|y\|_{\mathfrak{g}}=1}\left\|\left[x, y-\operatorname{Ad}_{k}^{-1} y\right]\right\|_{\mathfrak{g}} \\
& \left.\leq \sqrt{2-2 \cos |k|_{G}} \sup _{\|y\|_{\mathfrak{g}}=1}\|[x, y]\|_{\mathfrak{g}}+|x|_{\mathfrak{g}} \sup _{\|y\|_{\mathfrak{g}}=1} \| y-\operatorname{Ad}_{k}^{-1} y\right] \|_{\mathfrak{g}} \\
& \leq 2 \sqrt{2-\cos |k|_{G}|x|_{\mathfrak{g}}} \\
& =C|x|_{\mathfrak{g}} \\
& <C^{n+1} \frac{\pi}{2},
\end{aligned}
$$

as desired.

It follows that the operator norms of the sequence $\left\{h_{n}\right\}$ converge to zero. Therefore the sequence $\left\{h_{n}\right\}$ converges to the identity of $G$. Since $H$ is a discrete subgroup, it follows that $h_{n}$ is equal to the identity if $n$ is sufficiently large. We will next show that $h_{n}=1$ for all $n>0$, using an argument of Frobenius which proceeds by a descending induction on $n$. Once we know that $h_{1}=1$, the proof will be complete. Assume that $h_{n+1}=1$. Then $k$ commutes with $h_{n}$, and therefore also with $h_{n} k=h_{n-1} k h_{n-1}^{-1}$. It follows that $\mathfrak{g} \otimes_{\mathbb{R}} \mathbb{C}$ admits a basis 
whose elements are eigenvectors for both $k$ and $h_{n-1} k h_{n-1}^{-1}$. If the eigenvalues are the same in both cases, then we deduce that $k=h_{n-1} k h_{n-1}^{-1}$, so that $h_{n}$ is the identity and we are done. Otherwise, there exists $v \in \mathfrak{g} \otimes_{\mathbb{R}} \mathbb{C}$ which is an eigenvector for both $k$ and $h_{n-1} k h_{n-1}^{-1}$, with different eigenvalues. Equivalently, both $v$ and $h_{n-1} v$ are eigenvectors for $k$, with different eigenvalues. Thus $v$ and $h_{n-1} v$ are orthogonal, which implies $\left|h_{n-1}\right|_{G} \geq \frac{\pi}{2}$, a contradiction.

\section{The Proof when $H$ is Discrete}

In this section, we will give the proof of Theorem 1 in the case where $H$ is a discrete subgroup. The idea is to show that if $G / H$ is too small, then $H$ contains noncommuting elements which are close to the identity, contradicting Theorem 6 . In the statements that follow, we let $\alpha$ denote the smallest positive real solution to $\cos (\alpha)=\frac{7}{8}$ and $\beta$ the smallest positive real solution to the transcendental equation $\cos ^{2}(\alpha-\beta)+\sin ^{2}(\alpha-\beta) \cos \left(\frac{\pi}{2}-\beta\right)=\cos (4 \beta)$.

Lemma 7. Let $G$ be a compact, connected Lie group with trivial center. Then there exist elements $h, k \in G$ having the property that for any $h^{\prime}, k^{\prime} \in G$ with $d_{G}\left(h, h^{\prime}\right), d_{G}\left(k, k^{\prime}\right)<\beta$, we have $\left|h^{\prime}\right|_{G}<\frac{\pi}{2},\left|k^{\prime}\right|_{G}<\alpha$, and $\left[h^{\prime}, k^{\prime}\right] \neq 1$.

Proof. Choose a (local) embedding $p: S U(2) \rightarrow G$ corresponding to a root of some simple component of $G$. We will assume that if the relevant component has roots of two different lengths, then the embedding $p$ corresponds to a long root. This ensures that the weights of $S U(2)$ acting on $\mathfrak{g}$ are no larger than the weights of the adjoint representation. In the Lie algebra $\mathfrak{s o}(3)$ of $S U(2)$, we let $x$ and $y$ denote infinitesimal rotations of angles $\frac{\pi}{2}-\beta$ and $\alpha-\beta$ about orthogonal axes. Then, by the above condition on weights, we deduce that $h=p(\exp (x))$ and $k=p(\exp (y))$ satisfy the conditions $|h|_{G}=\frac{\pi}{2}-\beta,|k|_{G}=\alpha-\beta$. We claim that the pair $h, k \in G$ satisfies the conclusion of the lemma. To see this, choose any pair $h^{\prime}, k^{\prime} \in G$ with $d\left(h, h^{\prime}\right), d\left(k, k^{\prime}\right)<\beta$. Then we deduce $\left|h^{\prime}\right|_{G}<\frac{\pi}{2}$, $\left|k^{\prime}\right|_{G}<\alpha$ from the triangle inequality. To complete the proof, we must show that $h^{\prime}$ and $k^{\prime}$ do not commute. To see this, we let $v$ denote the image in $\mathfrak{g}$ of a vector in $\mathfrak{s o}(3)$ about which $x$ is an infinitesimal rotation. Then $h v=v$, while $\angle(v, k v)=\alpha-\beta$. Elementary trigonometry now yields

$$
\begin{aligned}
\angle(h k v, k h v) & =\angle(h k v, k v) \\
& =\cos ^{-1}\left(\cos ^{2}(\alpha-\beta)+\sin ^{2}(\alpha-\beta) \cos \left(\frac{\pi}{2}-\beta\right)\right) \\
& =\cos ^{-1}(\cos (4 \beta))=4 \beta .
\end{aligned}
$$

By the triangle inequality, we get

$$
\begin{aligned}
4 \beta= & \angle(h k v, k h v) \\
\leq & \angle\left(h k v, h^{\prime} k v\right)+\angle\left(h^{\prime} k v, h^{\prime} k^{\prime} v\right)+\angle\left(h^{\prime} k^{\prime} v, k^{\prime} h^{\prime} v\right) \\
& +\angle\left(k^{\prime} h^{\prime} v, k^{\prime} h v\right)+\angle\left(k^{\prime} h v, k h v\right) \\
< & 4 \beta+\angle\left(h^{\prime} k^{\prime} v, k^{\prime} h^{\prime} v\right),
\end{aligned}
$$

which implies $\angle\left(h^{\prime} k^{\prime} v, k^{\prime} h^{\prime} v\right)>0$ so that $\left[h^{\prime}, k^{\prime}\right] \neq 1$.

We can now complete the proof of Theorem 1 in the case where $H$ is discrete: 
Proof. Choose $h, k \in G$ satisfying the conclusion of Lemma 7. Since $G / H$ has diameter less than $\beta$, the cosets $h H$ and $k H$ are within $\beta$ of the identity coset in $G / H$, which implies that there exist $h^{\prime}, k^{\prime} \in H$ with $d\left(h, h^{\prime}\right), d\left(k, k^{\prime}\right)<$ $\beta$. Lemma 7 ensures that $h^{\prime}$ and $k^{\prime}$ do not commute, which contradicts Theorem 6 .

\section{The Proof when $G$ is Simple}

In this section, we give the proof of the main theorem in the case where $H$ is nondiscrete and $G$ is simple. The idea in this case is to show that because the Lie algebra $\mathfrak{h}$ of $H$ cannot be a $G$-invariant subspace of $\mathfrak{g}$, the action of $G$ automatically moves it quite a bit: this is made precise by Theorem 10 . Since $\mathfrak{h}$ is invariant under the action of $H$, this will force $G / H$ to have large diameter in the operator norm. We begin with some general remarks about angles between subspaces of a Hilbert space. Let $V$ be a real Hilbert space, and let $U, W \subseteq V$ be linear subspaces. The angle $\angle(U, W)$ between $U$ and $W$ is defined to be

$$
\left.\max \left(\sup _{u \in U-\{0\}} \inf _{w \in W-\{0\}}|\angle(u, w)|, \sup _{w \in W-\{0\}} \inf _{u \in U-\{0\}} \mid \angle(u, w)\right) \mid\right) .
$$

Note that for a fixed unit vector $u \in U$, the cosine of the minimal angle $\angle(u, w)$ with $w \in W$ is equal to the length of the orthogonal projection of $u$ onto $W^{\perp}$. Thus, the sine of the minimal (positive) angle is equal to the length of the orthogonal projection of $u$ onto $W^{\perp}$. Consequently we have

$$
\sin \left(\sup _{u \in U-\{0\}} \inf _{w \in W-\{0\}}|\angle(u, w)|\right)=\sup _{\|u\|=1,\left\|w^{\perp}\right\|=1}\left\langle u, w^{\perp}\right\rangle
$$

which is symmetric in $U$ and $W^{\perp}$. From this symmetry we can deduce:

Lemma 8. For any pair of subspaces $U, W \subseteq V$, the angle $\angle(U, W)$ is equal to the angle $\angle\left(U^{\perp}, W^{\perp}\right)$.

We will also need the following elementary fact:

Lemma 9. Let $V$ be a finite-dimensional Hilbert space, and let $A$ be an endomorphism of $V$ having rank $k$. Then $|\operatorname{Tr}(A)| \leq k|A|$.

Proof. Choose an orthonormal basis $\left\{v_{i}\right\}_{1 \leq i \leq n}$ for $V$ having the property that $A v_{i}=0$ for $i>k$. Then

$$
|\operatorname{Tr}(A)|=\left|\sum_{i}\left\langle v_{i}, A v_{i}\right\rangle\right| \leq \sum_{1 \leq i \leq k}\left|\left\langle v_{i}, A v_{i}\right\rangle\right| \leq \sum_{1 \leq i \leq k}|A|=k|A|
$$

We now proceed to the main point.

Theorem 10. Let $G$ be a compact Lie group acting irreducibly on a (necessarily finite dimensional) complex Hilbert space $V$. Let $W \neq 0, V$ be a nontrivial subspace. Then there exists $g \in G$ such that $\angle(W, g W) \geq \frac{\pi}{4}$. 
Proof. Suppose, to the contrary, that $\angle(W, g W)<\frac{\pi}{4}$ for all $g \in G$. Let $V$ have dimension $n$. Replacing $W$ by $W^{\perp}$ if necessary, we may assume that the dimension $k$ of $W$ satisfies $k \leq \frac{n}{2}$. For any subspace $U \subseteq V$, we let $\Pi_{U}$ denote the orthogonal projection onto $U$. For each $g \in G$, projection from $g W$ onto $W^{\perp}$ or from $W^{\perp}$ to $g W$ shrinks lengths by a factor of $\sin \angle(W, g W) \leq \sin \frac{\pi}{4}$ at least. It follows that

$$
\left|\Pi_{W^{\perp}} \Pi_{g W} \Pi_{W^{\perp}}\right| \leq\left|\Pi_{W^{\perp}} \Pi_{g W}\right|\left|\Pi_{g W} \Pi_{W^{\perp}}\right|<\frac{1}{2} .
$$

Using the identity $\operatorname{Tr}(A B)=\operatorname{Tr}(B A)$, we deduce

$$
\begin{aligned}
\operatorname{Tr}\left(\Pi_{g W} \Pi_{W^{\perp}}\right) & =\operatorname{Tr}\left(\Pi_{g W} \Pi_{W^{\perp}} \Pi_{W^{\perp}}\right) \\
& =\operatorname{Tr}\left(\Pi_{W^{\perp}} \Pi_{g W} \Pi_{W^{\perp}}\right) \leq k\left|\Pi_{W^{\perp}} \Pi_{g W} \Pi_{W^{\perp}}\right| \\
& <\frac{k}{2} .
\end{aligned}
$$

Integrating this result over $G$ (with respect to a Haar measure which is normalized so that $\int_{G} 1=1$ ), we deduce

$$
\operatorname{Tr}\left(\left(\int_{G} \Pi_{g W}\right) \Pi_{W^{\perp}}\right)=\int_{G} \operatorname{Tr}\left(\Pi_{g W} \Pi_{W^{\perp}}\right)<\frac{n}{2} .
$$

On the other hand, $\int_{G} \Pi_{g W}$ is a $G$-invariant element of $\operatorname{End}(V)$. Since $V$ is irreducible, Schur's lemma implies that $\int_{G} \Pi_{g W}=\lambda 1_{V}$ for some scalar $\lambda \in \mathbb{C}$. We can compute $\lambda$ by taking traces:

$$
\begin{aligned}
n \lambda & =\operatorname{Tr}\left(\lambda 1_{V}\right) \\
& =\operatorname{Tr}\left(\int_{G} \Pi_{g W}\right) \\
& =\int_{G} \operatorname{Tr}\left(\Pi_{g W}\right)=k,
\end{aligned}
$$

so that $\lambda=\frac{k}{n}$. Thus $\frac{k(n-k)}{n}=\operatorname{Tr}\left(\frac{k}{n} \Pi_{W}^{\perp}\right)<\frac{k}{2}$, so that $2(n-k)<n$, a contradiction.

From Theorem 10, one can easily deduce the analogous result in the case when $V$ is a real Hilbert space, provided that $V \otimes_{\mathbb{R}} \mathbb{C}$ remains an irreducible representation of $G$. Using this, we can easily complete the proof of Theorem 1 in the case where $G$ is simple and $H$ is nondiscrete (with an even better constant).

Proof. Let $\mathfrak{h}$ denote the Lie algebra of $H$. Since $H \neq G$ and $G$ is connected, $\mathfrak{h} \subsetneq \mathfrak{g}$. Since $H$ is nondiscrete, $\mathfrak{h} \neq 0$. Since $\mathfrak{g} \otimes_{\mathbb{R}} \mathbb{C}$ is an irreducible representation of $G$, we deduce that there exists $g \in G$ such that $\angle(g \mathfrak{h}, \mathfrak{h}) \geq \frac{\pi}{4}$. Now one deduces that for any $h \in H, g h^{\prime} \in g H$, the distance

$$
d\left(g h^{\prime}, h\right)=\left|g h^{\prime} h^{-1}\right|_{G} \geq \angle\left(g h^{\prime} h^{-1} \mathfrak{h}, \mathfrak{h}\right)=\angle(g \mathfrak{h}, \mathfrak{h}) \geq \frac{\pi}{4} .
$$

It follows that the distance between the cosets $g H$ and $H$ in $G / H$ is at least $\frac{\pi}{4}$. 


\section{The General Case}

We now know that Theorem 1 is valid under the additional assumption that the group $G$ is simple. We will complete the proof by showing how to reduce to this case. The main tool is the following observation:

Proposition 11. Let $\pi: G \rightarrow G^{\prime}$ be a surjection of compact connected Lie groups with trivial center, let $H$ be a closed subgroup of $G$ and $H^{\prime}=\pi(H)$ its image in $G^{\prime}$. Then $\operatorname{diam}\left(G^{\prime} / H^{\prime}\right) \leq \operatorname{diam}(G / H)$.

Proof. For any points $x^{\prime}, y^{\prime} \in G^{\prime} / H^{\prime}$, we can lift them to a pair of points $x, y \in$ $G / H$. It will suffice to show $d_{G / H}(x, y) \geq d_{G^{\prime} / H^{\prime}}\left(x^{\prime}, y^{\prime}\right)$. The left hand side is equal to

$$
\inf _{g x=y}|g|_{G}
$$

and the right hand side to

$$
\inf _{g^{\prime} x^{\prime}=y^{\prime}}\left|g^{\prime}\right|_{G^{\prime}}
$$

To complete the proof, it suffices to show that $|g|_{G} \geq|\pi(g)|_{G^{\prime}}$. This follows immediately since we may identify the Lie algebra $\mathfrak{g}^{\prime}$ of $G^{\prime}$ with a direct summand of $\mathfrak{g}$.

Now assume that $G$ is a compact, connected Lie group with trivial center. Then it is a product of simple factors $\left\{G_{\alpha}\right\}_{\alpha \in \Lambda}$. Let $\pi_{\alpha}: G \rightarrow G_{\alpha}$ denote the projection. Let $H \subsetneq G$ be a closed subgroup. If $\pi_{\alpha} H \neq G_{\alpha}$ for some $\alpha \in \Lambda$, then $\operatorname{diam}(G / H) \geq \operatorname{diam}\left(G_{\alpha} / \pi_{\alpha} H\right) \geq \beta$ and we are done. Otherwise, $\pi_{\alpha}$ induces a surjection of Lie algebras $\mathfrak{h} \rightarrow \mathfrak{g}_{\alpha}$ for each $\alpha$. By the structure theory of reductive Lie algebras, we deduce that $\mathfrak{h}=\mathfrak{h}_{\alpha} \oplus \mathfrak{k}_{\alpha}$, where $\pi_{\alpha}$ is zero on $\mathfrak{k}_{\alpha}$ and induces an isomorphism $\mathfrak{h}_{\alpha} \simeq \mathfrak{g}_{\alpha}$. Since $\mathfrak{h}_{\alpha}$ is therefore simple, $\mathfrak{k}_{\alpha}$ may be characterized as the centralizer of $\mathfrak{h}_{\alpha}$ in $\mathfrak{h}$. Since $H \neq G$ and $G$ is connected, $H$ must have smaller dimension than $G$. It follows that the subalgebras $\mathfrak{h}_{\alpha} \subseteq \mathfrak{h}$ cannot all be distinct. Choose $\alpha, \alpha^{\prime} \in \Lambda$ with $\mathfrak{h}_{\alpha}=\mathfrak{h}_{\alpha^{\prime}}$. The the map $H \rightarrow G_{\alpha} \times G_{\alpha^{\prime}}$ is not surjective on Lie algebras. Without loss of generality, we may replace $G$ by $G_{\alpha} \times G_{\alpha^{\prime}}$ and $H$ by its image in $G_{\alpha} \times G_{\alpha^{\prime}}$. Since the Lie algebra of $H$ now maps isomorphically onto the Lie algebras of the factors $G_{\alpha}$ and $G_{\alpha^{\prime}}$, it follows that the connected component $H_{0}$ of the identity in $H$ is isomorphic to $G_{\alpha}$, which is included diagonally in $G_{\alpha} \times G_{\alpha^{\prime}}$. Then $H=H_{0}\left(H \cap\left(G_{\alpha} \times 1\right)\right)$. The intersection $K=H \cap\left(G_{\alpha} \times 1\right)$ is normalized by $H_{0}=\left\{(g, g): g \in G_{\alpha}\right\}$, hence it is normalized by $G_{\alpha} \times\{e\}$. Since $G_{\alpha^{\prime}}$ is simple, we deduce that $K=\{e\}$. Thus $H=H_{0}$ is embedded diagonally in $G_{\alpha} \times G_{\alpha^{\prime}}$. We have already considered this case in Example 2, where we saw that the diameter of $G^{\prime} / H^{\prime}$ is at least $\frac{\pi}{2}$.

Remark 12. If we restrict our attention to the case where $H$ is a connected subgroup of $G$, then our proof gives a better lower bound of $\frac{\pi}{4}$. 


\section{References}

[NC] M. A. Nielsen, I. L. Chuang, Quantum computation and quantum information, Cambridge University Press, Cambridge, 2000.

Microsoft Research, One Microsoft Way, Redmond, WA 98052, U.S.A.

E-mail address: michaelf@microsoft.com

Caltech, 1200 East California Boulevard, Pasadena, CA 91125, U.S.A.

E-mail address: kitaev@iqi.caltech.edu

Mit, 77 Massachusetts Avenue, Cambridge, MA 02139-4307, U.S.A.

E-mail address: lurie@math.mit.edu 